\title{
Elemente und Ephemeriden des neuen Cometen 1883 Swift-Brooks.
}

Die Elemente sind erhalten aus den Beobachtungen:

1883 Febr. 24 Cambridge Mass.

März I Leipzig

$T=\mathrm{r}_{883}$ Febr. 18.8623 M. Z. Berlin

$\omega=110^{\circ} 36^{\prime} 24^{\prime \prime}$

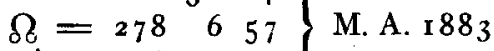

$i=78417$ )

$\log q=9.880^{8}$

Mittl. Beob. $\delta \lambda \cos \beta=-28^{\prime \prime}$

$$
\delta \beta=-6^{\prime \prime}
$$

Ephemeride für $\mathbf{1}^{\mathrm{h}}$ mittlere $\mathrm{Zt}$. Berlin.

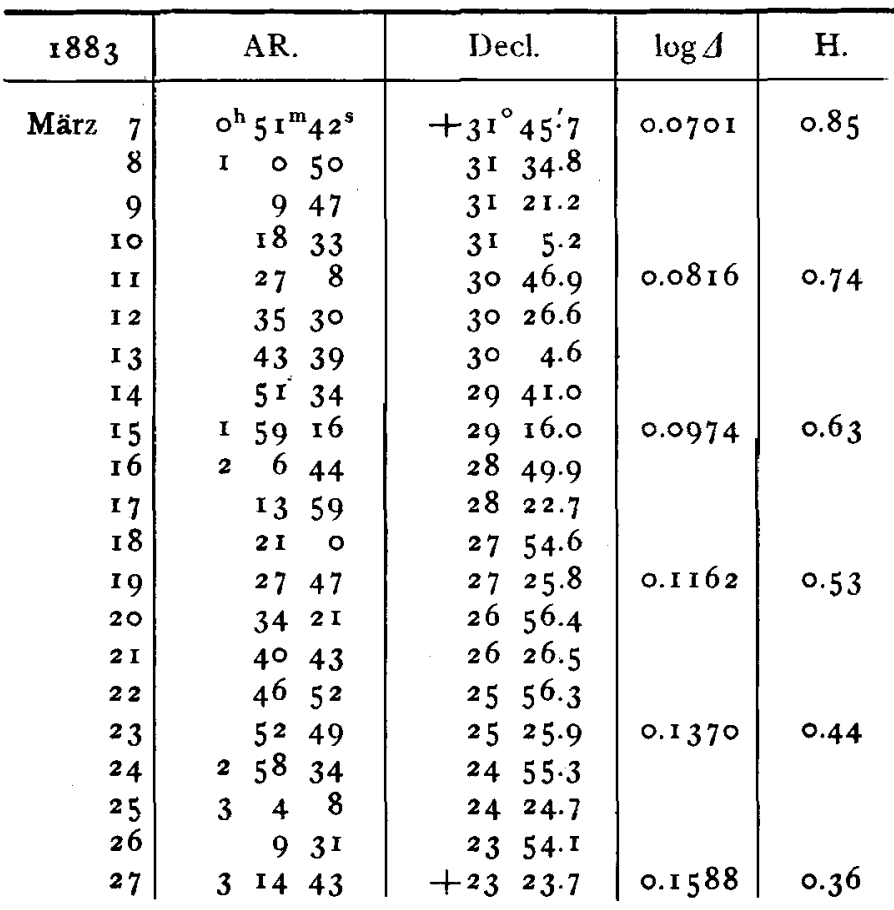

Helle bei der Entdeckung $=$ I gesetzt.

Berlin 1883 März 5.

Dr. H. Oppenheim.
Die der Rechnung zu Grunde liegenden Beobachtungen waren:

M.Z. $\quad$ app. $\delta$ app.

I. Febr. $24{ }_{1 I^{\mathrm{h}}} 5^{8^{\mathrm{m}}} 18^{\mathrm{s}}$ Greenw. $23^{\mathrm{h}} 7^{\mathrm{m}} 23^{\mathrm{s}} \cdot 2+30^{\circ} 27^{\prime} 44^{\prime \prime}$

2. $\quad 28 \quad 7$ Io 0 Palermo $2343 \quad 4.4$ I 3 I $37 \quad 47.8$

3. 》 $74222 》 234316.07 \quad 31386.0$

4. $\quad 7 \quad \begin{array}{lllllllllll}7 & 43 & 12 & \text { Rom } & 23 & 43 & 19.58 & 31 & 37 & 54.5\end{array}$

5. März $4 \quad 8 \quad 4$ I 48 Wien $\quad \begin{array}{llllllll} & 22 & 8.69 & 32 & 1 & 50.4\end{array}$

6. 84 I I 7 》 $8227.86+32$ I 37.2

Aus den Beobachtungen I, dem Mittel aus 2, 3, 4 und dem von 5, 6 erhielt ich mit Berüicksichtigung des

Einflusses von Parallaxe und Aberration folgendes parabolisches Elementen-System:

$$
\begin{aligned}
& T=\text { Febr. I8.97574 } \\
& \omega=\text { I } 10^{\circ} 53^{\prime} \text { II".8) } \\
& \left.\delta=\begin{array}{rrr}
278 & 7 & 0.8
\end{array}\right\} \text { Mittl. Aeq. } 1883.0 \\
& i=78 \quad 5 \text { I1.9 } \\
& \log q=9.880780
\end{aligned}
$$

\begin{tabular}{|c|c|c|c|c|}
\hline 1883 & AR. & Decl. & $\log 4$ & $\mathrm{H}$. \\
\hline März 26 & $3^{h} 9^{m} 33^{s}$ & $+23^{\circ} 50^{\prime} \cdot 3$ & 0.15301 & \\
\hline 27 & I 445 & $23 \quad 19.7$ & $0.15^{8} 53$ & \\
\hline 28 & 1947 & 2249.3 & 0.16408 & 0.345 \\
\hline 29 & 2440 & 22 I 9.1 & 0.16964 & \\
\hline 30 & 2924 & 2 I 49.2 & o. I 752 I & \\
\hline $3 I$ & 3359 & $21 \quad 19.6$ & 0.18078 & \\
\hline April I & $\begin{array}{ll}38 & 25\end{array}$ & $20 \quad 50.3$ & 0.18634 & 0.283 \\
\hline 2 & 4243 & $20 \quad 21.4$ & $0.19 \times 89$ & \\
\hline 3 & $4^{6} \quad 54$ & I9 52.9 & o.19742 & \\
\hline 4 & $5^{\circ} 5^{8}$ & $19 \quad 24.8$ & 0.20293 & \\
\hline 5 & 35455 & +1857.0 & $0.2084 \mathrm{I}$ & 0.233 \\
\hline
\end{tabular}

Darstellung der mittleren Beobachtung im Sinne $(B-R)$ :

$$
\Delta \lambda \cos \beta=+4.9 \quad \Delta \beta=+3.9 \text {. }
$$

Ephemeride für I $^{\text {h }}{ }^{\text {mittlere }} \mathrm{Zt}$. Berlin.

Bemerkung. Da die Ephemeride nahe mit Dr. Oppenheim's Rechnung stimmt, wird hier nur der Schluss derselben mitgetheilt.

Sternwarte Wien.

\section{In h a l t:}

Zn Nr. 2497. Wilhelm Schur. Bestimmung der geographischen Breite der Strassburger Sternwarte nach der Methode von Horrebow. I. - Fr. Schwab. Ueber den grossen September-Cometen 1882 II. 3. - Beobachtungen des neuen Cometen 1883 Swift-Brooks: Am Refractor der Kieler Sternwarte. I1. - Auf der Sternwarte des Collegio Romano in Rom. I I. - Auf der Sternwarte in Dresden. 13. - Auf der Sternwarte in Strassburg. 13. - Auf des Sternwarte in Nicolaiew. 13. - Auf der Sternwarte in Hamburg. 13. H. Oppenheim, F. $v$. Hepperger. Elemente und Ephemeriden des neuen Cometen 1883 Swift-Brooks. I 5. 